\title{
ON THE REDUCTION OF COMPLEX BORDISM TO UNORIENTED BORDISM ${ }^{1}$
}

\author{
DAVID COPELAND JOHNSON
}

\begin{abstract}
The image of the natural transformation from the complex bordism of a $\mathrm{CW}$ complex $X, M U_{*}(X)$, to its unoriented bordism, $N_{*}(X)$, is contained in a subgroup identified with $H_{*}(X ; Z) \otimes\left(N_{*}\right)^{2}$. A characterization is given for the CW complexes for which the image and the subgroup coincide.
\end{abstract}

Introduction. Let $M U_{*}(X)$ and $N_{*}(X)$ denote, respectively, the complex and the unoriented bordism of a CW complex $X$. For finite complexes, there are dual multiplicative cohomology theories $M U^{*}\left({ }_{-}\right)$and $N^{*}(-)$. There are natural forgetful transformations

$$
\phi_{*}(X): M U_{*}(X) \rightarrow N_{*}(X) \text { and } \phi^{*}(X): M U^{*}(X) \rightarrow N^{*}(X) \text {. }
$$

Let $j:\left(N^{*}\right)^{2} \rightarrow N^{*}$ denote the inclusion of the subring consisting of squares of elements of the coefficient ring of unoriented (co)-bordism, $N^{*} \cong N_{-*}$. Recall that $N_{*}$ has the structure of a graded $Z_{2}$ polynomial ring; so $j$ is a split monomorphism. Boardman and Quillen have given a natural multiplicative isomorphism of cohomology theories,

$$
\theta^{*}(X): N^{*}(X) \rightarrow H^{*}\left(X ; Z_{2}\right) \otimes_{Z_{2}} N^{*}
$$

([1], [6]). We have then a natural transformation of functors (the first functor does not give a cohomology theory).

$$
\begin{aligned}
\psi^{*}(X): H^{*}(X ; Z) \otimes\left(N^{*}\right)^{2} & \stackrel{\rho \otimes 1}{\longrightarrow} H^{*}\left(X ; Z_{2}\right) \otimes\left(N^{*}\right)^{2} \cong H^{*}\left(X ; Z_{2}\right) \otimes_{Z_{2}}\left(N^{*}\right)^{2} \\
& \stackrel{1 \otimes j}{\longrightarrow} H^{*}\left(X ; Z_{2}\right) \otimes_{Z_{2}} N^{*} \stackrel{\theta^{*}(X)}{\longleftarrow} N^{*}(X) .
\end{aligned}
$$

Here $\rho$ is reduction from integral to mod 2 cohomology. $\rho \otimes 1$ is monic as it appears as a factor of the following universal-coefficient-theorem monomorphism

$$
H^{*}(X ; Z) \otimes\left(N^{*}\right)^{2} \stackrel{\rho \otimes 1}{\longrightarrow} H^{*}\left(X ; Z_{2}\right) \otimes\left(N^{*}\right)^{2} \cong H^{*}\left(X ;\left(N^{*}\right)^{2}\right) .
$$

Received by the editors November 1, 1972.

AMS (MOS) subject classifications (1970). Primary 55B20, 57D75, 57D90; Secondary $18 \mathrm{G} 20$.

Key words and phrases. Complex bordism, unoriented bordism, projective dimension. ${ }^{1}$ Supported by NSF GP-33883.

(c) American Mathematical Society 1973 
So $\psi^{*}(X)$ gives a multiplicative, monic, natural transformation of functors. Dually, there is a natural monomorphism of functors

$$
\psi_{*}(X): H_{*}(X ; Z) \otimes\left(N_{*}\right)^{2} \rightarrow N_{*}(X) .
$$

Recall that $Z_{(2)}$ is the integers localized at the prime two (the subring of rationals represented by fractions with odd denominators). $Z_{(2)}$ is a flat abelian group and $M U_{*}(-) \otimes Z_{(2)}$ is a homology theory with an external multiplication.

Both homomorphisms $\phi_{*}(X)$ and $\psi_{*}(X)$ have range $N_{*}(X)$. When $X$ is a one point space, Milnor showed that their images coincide [4]. The purpose of this note is to record the following observation which provides a converse to and includes the result of [5].

TheOREM. For any CW complex $X$, image $\phi_{*}(X) \subset$ image $\psi_{*}(X)$, image $\phi_{*}(X)=$ image $\psi_{*}(X)$ if and only if the projective dimension of $M U(X) \otimes Z_{(2)}$ as a $M U_{*} \otimes Z_{(2)}$ module is at most one.

Of course, for finite complexes, the analogous statement relating the images of $\phi^{*}(X)$ and $\psi^{*}(X)$ is also true (but the reader should be warned that the projective dimensions of $M U_{*}(X) \otimes Z_{(2)}$ and of $M U^{*}(X) \otimes Z_{(2)}$ as $M U_{*} \otimes Z_{(2)} \cong M U^{-*} \otimes Z_{(2)}$ modules are not in general equal). Since $\psi^{*}(X)$ is monic and multiplicative, we obtain the following corollary which may be of interest.

COROLlary. For finite complexes, there is a natural multiplicative transformation of functors

$$
\psi^{*}(X)^{-1} \circ \phi^{*}(X): M U^{*}(X) \rightarrow H^{*}(X ; Z) \otimes\left(N^{*}\right)^{2} .
$$

$\psi^{*}(X)^{-1} \circ \phi^{*}(X)$ is epic if and only if the projective dimension of $M U^{*}(X) \otimes Z_{(2)}$ as a $M U^{*} \otimes Z_{(2)}$ module is at most one.

Proof of Theorem. Given a CW complex $X$, there is a -1 -connected CW spectrum $A$ with $H_{*}(A ; Z)$ free abelian and a stable map $f: A \rightarrow X$ such that the induced homomorphism in complex bordism $\left(f_{3}\right.$ in diagram $\left.(*)\right)$

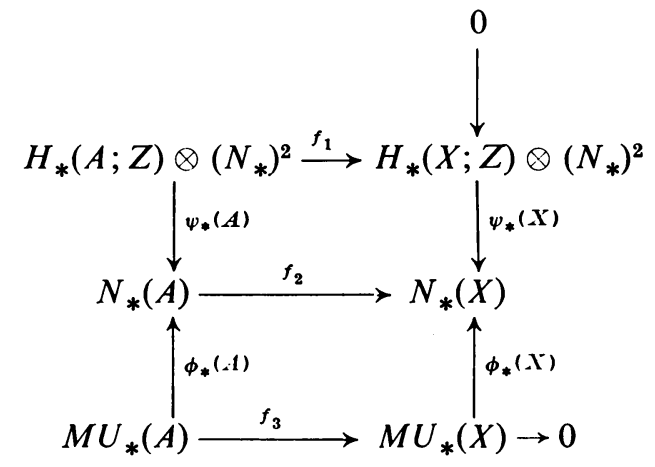


is epic ([2, Proposition 2.4 for the finite case]; [3, Lemma 5 for the generalization]).

From Milnor's result that image $\psi_{*}($ point $)=$ image $\phi_{*}$ (point) and from the fact that $H_{*}(A ; Z)$ is free abelian, it is routine to show that the images of $\psi_{*}(A)$ and $\phi_{*}(A)$ coincide. From the commutativity of $(*)$, we have image $\phi_{*}(X)=\phi_{*}(X) \circ f_{3}=f_{2}\left(\right.$ image $\left.\phi_{*}(A)\right)=f_{2}\left(\right.$ image $\left.\psi_{*}(A)\right)=$ image $\psi_{*}(X) \circ f_{1} \subseteq$ image $\psi_{*}(X)$. Since $\psi_{*}(X)$ is a monomorphism, image $\phi_{*}(X)=$ image $\psi_{*}(X) \circ f_{1}$ is precisely image $\psi_{*}(X)$ if and only if $f_{1}=H_{*}(f ; Z) \otimes\left(N_{*}\right)^{2}$ is epic. We may identify $Z_{2}$ as the zero component of the graded $Z_{2}$ vector space, $\left(N_{*}\right)^{2}$. We have a chain of equivalences:

$f_{1}=H_{*}(f ; Z) \otimes\left(N_{*}\right)^{2}$ is epic

$\Leftrightarrow H_{*}(f, Z) \otimes Z_{2}$ is epic

$\Leftrightarrow f_{4}=H_{*}\left(f, Z_{(2)}\right) \cong H_{*}(f ; Z) \otimes Z_{(2)}$ is epic

$\Leftrightarrow \mu(X) \otimes Z_{(2)}$ is epic in $(* *)$

$\Leftrightarrow$ the projective dimension of $M U_{*}(X) \otimes Z_{(2)}$ as a $M U_{*} \otimes Z_{(2)}$ module is at most one.

The first two of these equivalences follow from elementary algebra. The third is by consideration of the commutative diagram $(* *)$.

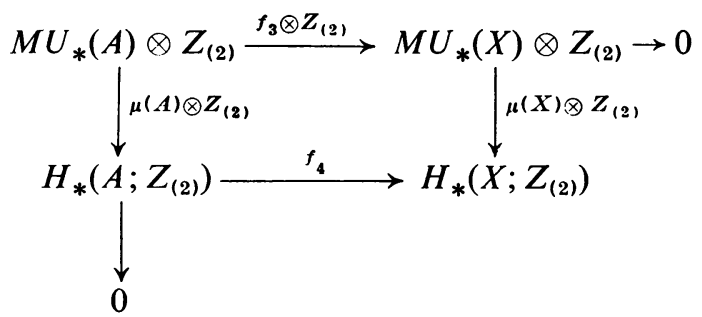

Since $H_{*}(A ; Z)$ is free abelian, the Thom homomorphism $\mu(A)$ is epic. The last equivalence is by Corollary $3.11 \otimes Z_{(2)}$ of [3] as extended in [2].

ACKnOWLedgement. The author thanks R. E. Stong for his forbearance. This note grew out of a spurious assertion made to him; it was motivated by A. Dold's review of [5] [Math. Rev. 43 (1972), 746. Abstract \#4039].

\section{REFERENCES}

1. T. Bröcker and T. tom Dieck, Kobordismentheorie, Lecture Notes in Math., vol. 178, Springer-Verlag, Berlin and New York, 1970. MR 43 \#1202.

2. P. E. Conner and L. Smith, On the complex bordism of finite complexes, Inst. Hautes Études Sci. Publ. Math. No. 37 (1969), 117-221. MR 42 \#2473.

3. P. S. Landweber, Complex bordism of classifying spaces, Proc. Amer. Math. Soc. 27 (1971), 175-179. MR 42 \#3782. 
4. J. Milnor, On the Stiefel-Whitney numbers of complex manifolds and of spin manifolds, Topology 3 (1965), 223-230. MR 31 \#5207.

5. G. E. Mitchell, The image of $\mathscr{U} *(X) \rightarrow \mathscr{N} *(X)$, Proc. Amer. Math. Soc. 26 (1970), 505-508. MR 43 \#4039.

6. D. Quillen, Elementary proofs of some results of cobordism theory using Steenrod operations, Advances in Math. 7 (1971), 29-56.

Department of Mathematics, University of Kentucky, LeXington, Kentucky 40506 